\title{
Correction: Application of an Adaptive, Digital, Game-Based Approach for Cognitive Assessment in Multiple Sclerosis: Observational Study
}

\author{
Wan-Yu Hsu ${ }^{1}$, PhD; William Rowles ${ }^{1}$, BA; Joaquin A Anguera ${ }^{1,2,3}$, PhD; Chao Zhao ${ }^{1}$, MSc; Annika Anderson ${ }^{1}$, BA; \\ Amber Alexander ${ }^{1}$, BSc; Simone Sacco ${ }^{1}$, MD; Roland Henry ${ }^{1}$, PhD; Adam Gazzaley ${ }^{1,2,3,4}, \mathrm{MD}$, PhD; Riley Bove ${ }^{1}$, \\ MD
}

${ }^{1}$ Department of Neurology, Weill Institute for Neurosciences, University of California, San Francisco, San Francisco, CA, United States

${ }^{2}$ Neuroscape, University of California, San Francisco, San Francisco, CA, United States

${ }^{3}$ Department of Psychiatry, University of California, San Francisco, San Francisco, CA, United States

${ }^{4}$ Department of Physiology, University of California, San Francisco, San Francisco, CA, United States

Corresponding Author:

Riley Bove, MD

Department of Neurology

Weill Institute for Neurosciences

University of California, San Francisco

675 Nelson Rising Lane

San Francisco, CA, 94158

United States

Phone: 14155952795

Email: riley.bove@ucsf.edu

\section{Related Article:}

Correction of: https://www.jmir.org/2021/1/e24356/

(J Med Internet Res 2021;23(1):e27440) doi: 10.2196/27440

In "Application of an Adaptive, Digital, Game-Based Approach for Cognitive Assessment in Multiple Sclerosis: Observational Study" (J Med Internet Res 2021;23(1):e24356) the authors noted two errors.

In the originally published manuscript, author Joaquin Anguera was incorrectly named. The following name has been included in the corrected version of the manuscript: Joaquin A Anguera.

In the originally published manuscript, a copyright credit was not included in the caption of Figure 1. The following text has been added to the end of the Figure 1 caption in the corrected version of the manuscript:

Copyright (C) 2020-2021, Akili Interactive Labs, Inc. All rights reserved.

The corrections will appear in the online version of the paper on the JMIR Publications website on January 27, 2021, together with the publication of this correction notice. Because this was made after submission to PubMed, PubMed Central, and other full-text repositories, the corrected article has also been resubmitted to those repositories.

This is a non-peer-reviewed article. Submitted 25.01.21; accepted 25.01.21; published 27.01.21.

Please cite as:

Hsu WY, Rowles W, Anguera JA, Zhao C, Anderson A, Alexander A, Sacco S, Henry R, Gazzaley A, Bove $R$

Correction: Application of an Adaptive, Digital, Game-Based Approach for Cognitive Assessment in Multiple Sclerosis: Observational Study

J Med Internet Res 2021;23(1):e27440

URL: http://www.jmir.org/2021/1/e27440/

doi: $\underline{10.2196 / 27440}$

PMID: 33502997 
(CWan-Yu Hsu, William Rowles, Joaquin A Anguera, Chao Zhao, Annika Anderson, Amber Alexander, Simone Sacco, Roland Henry, Adam Gazzaley, Riley Bove. Originally published in the Journal of Medical Internet Research (http://www.jmir.org), 27.01.2021. This is an open-access article distributed under the terms of the Creative Commons Attribution License (https://creativecommons.org/licenses/by/4.0/), which permits unrestricted use, distribution, and reproduction in any medium, provided the original work, first published in the Journal of Medical Internet Research, is properly cited. The complete bibliographic information, a link to the original publication on http://www.jmir.org/, as well as this copyright and license information must be included. 